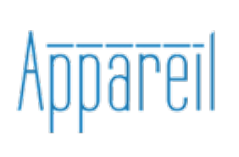

Appareil

$23 \mid 2021$

Poïétique du jeu vidéo

\title{
L'artiste pantocrator : le créateur de jeu vidéo au travail
}

\section{Pascal Krajewski}

\section{OpenEdition}

1 Journals

\section{Édition électronique}

URL : http://journals.openedition.org/appareil/4164

DOI : 10.4000/appareil.4164

ISSN : 2101-0714

Éditeur

MSH Paris Nord

Référence électronique

Pascal Krajewski, «L'artiste pantocrator : le créateur de jeu vidéo au travail », Appareil [En ligne], 23 | 2021, mis en ligne le 31 mars 2021, consulté le 02 avril 2021. URL : http://journals.openedition.org/ appareil/4164; DOI : https://doi.org/10.4000/appareil.4164

Ce document a été généré automatiquement le 2 avril 2021.

\section{(c) (i) (3)}

Appareil est mis à disposition selon les termes de la Licence Creative Commons Attribution - Pas d'Utilisation Commerciale - Pas de Modification 4.0 International. 


\title{
L'artiste pantocrator : le créateur de jeu vidéo au travail
}

\author{
Pascal Krajewski
}

\section{NOTE DE L'AUTEUR}

\section{Remerciements}

Ce texte a bénéficié d'interviews de concepteurs de jeux indépendants, croisés lors du festival Toulouse Game Dev en janvier 2020 :

Léonard Bertos : https://sites.google.com/view/leontroglodytefr/accueil

Carlos Botella : https://www.facebook.com/SpaceGameStudio

Benoît Jurado : https://akitl.itch.io/

\section{La place du spect-acteur}

1 Majoritairement, le régime des œuvres d'art est le suivant : un objet déterminé est reçu par un spectateur qui est libre de l'interpréter à sa guise, si possible dans l'horizon proposé par l'auteur (consciemment ou non). C'est un régime que l'on pourrait qualifier de factuel et interprétatif. Le régime des jeux vidéo, comme de toute œuvre interactive, est différent : un programme source, gros d'une multiplicité de possibles, est activé par un "spect-acteur» qui décide en partie de la forme que prendra son expérience interactive privée. C'est un régime virtuel et actualisé. Envisageons un instant que ce second régime soit compatible avec l'idée d'art, autrement dit qu'un art interactif soit possible.

2 Le régime traditionnel de l'œuvre d'art suppose un spectateur placé à la troisième personne, extérieur à l'œuvre. Il n'est, certes, pas passif face à cette dernière, puisqu'il la découvre et la comprend de façon singulière, en fonction de son histoire personnelle et de sa disponibilité du moment; mais il n'intervient pas dans la forme prise par l'œuvre. L'œuvre d'art y est un spectacle exogène, formellement déterminé, à 
découvrir, à faire résonner, à faire sien, mais toujours depuis une position extérieure. CEuvre et spectateur sont sur deux plans distincts. La relation " objet d'art-spectateur " est même paradigmatique d'une relation existentielle entre l'objet et le sujet: le sujet pose l'objet en face de lui en observant la chose du dehors, et, ce faisant, se constitue en sujet raisonnable et sensible.

Or, le régime nouveau de l'art interactif, et spécialement du jeu vidéo, suppose, lui, un spect-acteur à la première personne, partiellement intégré aux mécanismes de production formelle de l'œuvre, co-déterminant sa manifestation, c'est-à-dire son actualisation parmi un ensemble de possibles prédéterminés par le programme qui contient virtuellement tous les déploiements de l'œuvre. L'œuvre est une expérience vécue de l'intérieur; œuvre et spect-acteur s'hybrident pour donner forme à une actualisation particulière, fruit des puissances calculatoires de l'œuvre appareillée et de la relation nouée avec son interacteur. Objet et sujet ne se font plus face, ils nouent des interrelations, se répondent et se nourrissent à chaque instant, se co-déterminent.

Passer d'un objet d'art à percevoir à la troisième personne à un objet interactif à activer à la première personne va impliquer des changements drastiques dans les attendus esthétiques. Toute tentative autocritique de l'art contemporain considérée, la peinture de la Renaissance et ses théories esthétiques restent d'actualité pour l'œuvre factuelle : l'œuvre est une fenêtre ouverte sur un autre monde, elle est là pour en rendre compte, sur les modes très rationnels et perceptuels de la représentation et de l'expression ${ }^{1}$. Dans sa théorie esthétique, Theodor Adorno retient deux principes clés au bon fonctionnement d'une œuvre d'art: l'apparence et l'expression. L'apparence (Erscheinung) est pour lui l'accomplissement mimétique propre de l'œuvre d'art, une « ressemblance à soi ${ }^{2} »-$ autoreprésentation, pourrait-on dire ${ }^{3}-$, et l'expression est « le visage plaintif des œuvres ", « le non-subjectif dans le sujet, moins son expression que son empreinte $\rrbracket^{4}$. L'objet d'art factuel est apparence, il se représente en représentant un objet, une situation, un mouvement, une sensation - et il exprime non subjectivement un sentiment, un sens, une émotion, une révolte, une force, une colère, etc. En effet, la nature de l'objet d'art traditionnel est de se manifester uniment à un spectateur.

5 À partir du moment où l'œuvre d'art doit être vécue à la première personne, il faut pouvoir insérer le spectateur dans la représentation proposée et l'installer dans l'expression recherchée. Tout spectateur, avec sa singularité, ses envies, ses besoins. La représentation devra donc être labile, fluide, tandis que l'expression s'instillera par une sensibilisation physique du corps de l'«inter-acteur». C'est donc moins la représentation (et ses qualités pures, visuelles ou narratives) qui primera que la simulation (d'un monde, d'une action, d'un possible); et l'expression de l'œuvre laissera place à l'inspiration de l'interacteur (par un sentiment, une sensation). La représentation fait référence, dénote une situation, réelle ou imaginaire, qu'elle détermine entièrement; la simulation génère un monde fictif sui generis, dont elle ne dévoile chaque fois qu'un fragment de vie. L'expression est une propriété de l'objet ${ }^{5}$; l'inspiration s'intéresse exclusivement aux effets induits dans la chair et l'esprit du spectateur et consiste à instiller des sensations et des émotions par des moyens classiques (les qualités esthétiques de l'objet) et interactifs (l'excitation des sens du spect-acteur). Inspiration et simulation sont donc la continuation hypermoderne, technologique, virtuelle des deux piliers esthétiques de l'objet d'art factuel, l'expression et la représentation. Une telle descendance pourrait raisonnablement être 
considérée comme une possible continuation du rôle et des qualités de l'art. En se technologisant, cette nouvelle œuvre d'art se renie peut-être moins comme art qu'elle ne se complique. Aux régimes sémiotique, sémantique et symbolique de la représentation et de l'expression, va venir s'ajouter une dimension pragmatique qui intègre le spect-acteur dans l'œuvre, lui fait quitter sa place exogène de troisième personne pour lui faire endosser celle, à la première personne, de co-créateur endogène.

6 Ce changement de place du spectateur, de la troisième à la première personne, dans la manifestation de l'œuvre d'art voulue par un artiste tiers, nous semble fondamental du changement corrélatif qui doit s'opérer dans la création de l'œuvre. L'art de l'artiste interactif sera de simuler un monde et d'inspirer un corps interacteur. Ses outils spécifiques seront technologiques et informatiques, ce qui augure une nouvelle forme de pensée créatrice.

7 D'un point de vue objectal, on pourrait considérer que le jeu vidéo est l'une de ces sortes d'art interactif, fameuse car largement répandue auprès du public. Il est sans doute, d'abord et avant tout, un art interactif de masse. Son syntagme même ne doit pas nous tromper : il est parfois moins ludique qu'interactif (la partie de Solitaire que l'on lance sur un ordinateur lorsque l'ennui nous gagne ne nous désennuie pas vraiment; le joueur qui, échouant cinquante fois sur un passage ardu, se rend fou de rage, éprouvet-il un plaisir ludique ?) ; et il est parfois moins visuel que fonctionnel : il existe des jeux purement textuels, de même que l'épure visuelle peut suffire à accrocher l'œil et provoquer l'addiction, comme l'a démontré la longévité d'un Pong (Nolan Bushnell et Allan Alcorn, 1972). S'il n'est pas essentiellement ludique ni visuel, le jeu vidéo est essentiellement interactif. S'il est ludique, il n'en est pas nécessairement non artistique. S'il est artistique, ses enjeux créatifs propres se rattacheront à ces deux voies : simuler et inspirer.

\section{L'art de la simulation}

En quoi consiste cet art de la simulation propre au jeu vidéo ? En quoi se départit-il de l'art de la représentation propre aux arts plus classiques?

8 En tant qu'art technologique, le jeu vidéo fonde sur les puissances calculatoires des ordinateurs et les richesses appareillées des interfaces des capacités d'invention de formes sans précédent $^{6}$. Les formes visuelle et sonore, d'abord, sont des formes synthétiques dont les qualités se sont tellement améliorées en quelques décennies qu'on peut leur prédire un avenir impeccable: son enveloppant naturel, images hyperréalistes, mouvements fluides, etc. Simuler consistera d'abord à produire des univers phénoménologiques irréprochables pour la sensibilité humaine, une invention des apparences ${ }^{7}$ inouïe. L'image représentante, statique, déterminée et parachevée se voit troquée contre une image simulée, dynamique, sans cesse recalculée et réaffichée, jamais figée car intégrant les éléments d'un contexte variable.

Ce contexte est largement objectivé. L'image technologique n'est pas faite de touches de lumière ou de couleur, mais d'apparitions d'objets selon un certain point d'observation. L'image n'est pas créée une fois pour toutes, posant l'apparaître d'objets depuis un certain point d'observation (ce qu'accomplit la perspective), c'est l'essence des objets qui est codée afin de pouvoir fournir autant d'affichages que de points de vue (ce que réalisent les moteurs 3D). Il n'y a pas d'images, il n'y a qu'une pléiade d'objets, 
définis en leur taille, poids, couleurs, texture, résistance, emplacement relatif, etc. L'apparence finale n'est pas du ressort du créateur, mais de l'interface de visualisation. Certes, le créateur technologique passe son temps à vérifier le rendu de sa configuration objectale selon des points de vue privilégiés, mais ce n'est pas cette vision finale qu'il corrige, c'est le code qui détermine l'agencement des objets numériques. De plus, ces objets ne servent pas seulement comme motifs ponctuels dans l'image, mais comme ingrédients de l'image globale : l'effet qu'ils ont sur la lumière qui s'y reflète ou sur le son qu'ils étouffent, influence toute l'image produite.

Car, c'est là la troisième composante de cette figuration synthétique, elle est modélisée : elle intègre en son sein des approches et des dimensions processuelles et génétiques. Elle est autant connue dans son apparence et son essence que dans son énergie (ou plus exactement sa dynamis ${ }^{8}$ ), par son paraître instantané que par l'équation qui définit son déploiement temporel. La " physique " propre à chaque jeu est l'aspect le plus important et le moins visible d'un travail inépuisable (on pourra toujours aller plus loin dans la modélisation d'effets de réel) : on peut modéliser la gravité, l'effet du contact des objets, celui des chocs, le lancer de diverses armes, les brumes, les fumées, les reflets, la fatigue, etc. La copie du créateur est vierge, charge à lui de tout créer... Il ne copie pas les processus du réel, il invente les siens en les codant. Cela signifie qu'une telle image intègre en son sein une certaine vitalité. Sous l'influence du contexte de l'interaction, du milieu que forment les différents éléments de l'univers, et des effets ambiants codés comme des processus mathématiques, l'image technologique est vivace! Le son et l'image perçus par le spect-acteur dépendront de sa place, son orientation, de l'heure, la météo, les objets environnants - pour ne citer que quelques éléments objectifs de l'univers alentour. À ce stade, on pourrait dire que la simulation est une représentation synthétique objectivée, doublée d'effets de réel modélisés.

Telle est la réalité de terrain de tout artiste technologique. Il nous semble que l'œuvre vidéoludique ajoute de nouveaux traits à cet état de fait, traits qu'il nous faut aller chercher non plus dans la généalogie plastique de la discipline mais dans son obédience narrative.

11 Car, de même que l'on pouvait dire que l'image picturale classique "représente ", on pourra aussi soutenir que le discours romanesque «représente » une histoire, des personnages, des caractères et des évolutions. La représentation fictionnelle est la mise en forme déterminée, balisée et orientée d'un morceau de vie d'un tiers fictif, que l'on suit du dehors. Le créateur vidéoludique relève à nouveau plusieurs paris pour proposer une simulation de vécus, à expérimenter de l'intérieur...

12 La première force du langage narratif de jeu vidéo tient à sa capacité à sortir du modèle linéaire de la trame narrative. Le déroulement de l'action n'est pas (disons : peut ne pas être) linéaire, fixé à l'avance. Cette liberté d'action tient soit à la capacité de mouvements du protagoniste (ici le joueur), soit à sa latitude devant l'enchevêtrement de quêtes secondaires, soit à l'ouverture totale du monde proposé. Les mondes ouverts présentent là un sommet du genre. Les développeurs proposent un vaste jeu souvent multijoueur dans lequel les participants sont invités à errer à leur guise, agrémenté de quêtes locales qu'ils sont libres de suivre ou non, englobé dans un fil narratif qu'ils sont susceptibles d'ignorer, le tout en perpétuelle évolution en fonction des avancées des personnages non-joueurs, des désirs exprimés des joueurs, des volontés propres des créateurs et des financements disponibles! Simuler une histoire vidéoludique (pour les jeux qui en ont), c'est, dans un univers déjà créé, jalonner le chemin du joueur d'actions 
et d'informations directives qui le conduiront à travers un maquis d'étapes possibles vers l'une des fins préfigurées.

La simulation des actions relève de deux ordres. D'une part se trouvent les actions possibles et la gestique propre et souvent évolutive de l'avatar, dont les capacités s'accroissent ou s'adaptent avec le contexte narratif qu'il affronte (puissance des armes, capacité à voler ou escalader) - et, en regard, celles des objets ou personnages antagonistes. Cela signifie que les avatars sont associés à des caractéristiques quantifiées, sont reliés à la physique du monde et souvent soutenus par une aide tacite de la machine (les mouvements réalisés par le joueur peuvent être fébriles, son avatar réussira son geste sans férir). D’autre part sont conçus les épisodes directifs, étapes souvent nécessaires dans le déroulement d'un des fils narratifs proposés, qui trouvent dans la cinématique un moyen privilégié d'expression et souvent de célébration. Le créateur n'écrit donc pas une histoire comme le romancier, mais il tisse un réseau souvent très dense d'épisodes reliés entre eux, qu'il reviendra au joueur d'activer dans un ordre ouvert pour qu'il écrive son aventure personnelle.

La simulation des caractères, enfin, est peut-être la voie la plus récente. Les personnages non joueurs seraient des objets s'ils ne bénéficiaient de traits supplémentaires qui leur insufflent une caractérisation plus forte. Certes, ils sont d'abord et avant tout des vecteurs d'action (combat, orientation) et d'information (dialogues), qu'ils prodiguent en fonction de l'avancée du joueur. Ce sont des objets numériques renforcés de caractéristiques physiques, d'un comportement global et d'une batterie de dialogues qu'ils seront amenés à réciter ou non; mais ce sont aussi des nœuds privilégiés d'émotion et de sentiment. Le personnage est potentiellement un puits d'émotion pour lequel l'avatar rentre en sympathie: cette émotion pourra transparaître dans un mouvement de visage, un dialogue, une action partagée, un échange intime. Son comportement est aussi le reflet de celui du joueur : il pourra être ami ou ennemi, indifférent ou aidant selon l'état de l'avatar du joueur ou le choix que ce dernier fera dans son interaction sociale - et le vieux bougon que vous avez froissé en oubliant de frapper à sa porte pourra se dérider si vous tondez sa pelouse, sauf si vous avez eu le malheur de faucher ses tulipes! Les personnages non joueurs, bien que de nature numérique proche de celle des objets, ont des valeurs ludiques et narratives tout autres, notamment du fait de cette sympathie qui nous lie à eux, cette sympathie qui est celle-là même que les arts narratifs investissent depuis des siècles. Là où la représentation narrative visait en partie à stimuler la sympathie personnage-lecteur, la simulation vidéoludique ambitionnera plutôt d'activer une empathie avatar-joueur, notamment en cherchant à recréer des liaisons sympathiques entre l'avatar et les autres personnages.

15 Tels sont, nous semble-t-il, les deux paris de la simulation que le créateur de jeu vidéo doit relever: non pas représenter une scène, mais simuler un monde, c'est-à-dire synthétiser un univers cohérent d'apparence réaliste, dans lequel s'insuffle une vitalité processuelle; non pas narrer une tranche de vie, mais simuler des instants de vécu, c'est-à-dire plonger l'avatar du joueur dans une trame narrative cohérente, ouverte quoique dirigée, offrant une panoplie de caractères riches et réalistes. Loin de se contenter de produire un espace visuel ou un chemin narratif, elle propose un monde habité dans lequel le joueur peut déambuler et agir à sa guise, elle invente un authentique Lebenswelt : à la fois un monde du vivant (Lebens) et un monde du vécu ${ }^{9}$ (Erlebnis). In fine, c'est une véritable simulation de la vie et de son cours qui se dessine, 
en tout cas qui est posée comme l'idéal de création de l'artiste vidéoludique. L'art de la simulation vidéoludique consistera donc à simuler un univers et une trame, pour en faire un monde habité que le joueur peut expérimenter. Le monde du jeu vidéo parle de world building, la construction d'un monde, au sens plein du terme, donc.

\section{L'inspiration au cœur du gameplay}

16 "L'art est expressif lorsqu'il exprime quelque chose d'objectif subjectivement médiatisé : tristesse, énergie, désir ${ }^{10} »$. Classiquement, l'artiste cherche à exprimer quelque chose par son œuvre, selon une certaine modalité médiante, de telle sorte que cette chose puisse être reconnue par le récepteur. Il n'est pas si simple de se sentir ému par une musique culturellement éloignée : il est peu probable qu'un spectacle de nō fasse fondre en larmes un Occidental novice, et encore moins que le légendaire Tansen eût trouvé dans une cour européenne la place de choix qu'il avait chez le Grand Moghol. Les artistes se sont évertués pendant des siècles à faire en sorte que leurs œuvres s'expriment proprement, c'est-à-dire singulièrement.

17 À partir du moment où le spectateur est intégré dans le déploiement de l'œuvre, l'artiste peut chercher sa finalité non dans l'expression rattachée à l'objet d'art, mais directement dans l'inspiration qu'il veut produire chez le récepteur. Il lui importe moins de travailler à ce que son œuvre exprime quelque chose en soi, que de tester si son œuvre inspire bien quelque chose chez le joueur. Ce seront moins les qualités objectives de l'œuvre qui seront en ligne de mire que les effets produits chez le spectacteur. Bien sûr, celles-là mènent à ceux-ci, mais le créateur peut enfin agir selon son but : in-spirer des sentiments au joueur, littéralement "souffler dans", " animer par un souffle».

18 Le jeu vidéo possède pour parvenir à ce résultat deux fondements mécaniques spécifiques: l'implication du spect-acteur dans son déploiement et des capacités à induire des sensations physiques sur le joueur. Un bon jeu vidéo est sensationnel, il capte le joueur, le prend dans ses schèmes morphodynamiques ${ }^{11}$ et ludiques. La notion de game feel est peut-être la plus synthétique pour appréhender cela ${ }^{12}$ : ce "sentiment de jeu » qui prend et surprend le joueur, captif ravi et incrédule de sa propre excitation, ou, pour le dire dans les mots de l'un de ses théoriciens, Steve Swink :

[...] le sens tactile et kinesthésique de la manipulation d'objets virtuels. C'est la sensation de contrôle dans un jeu. [...] une sorte de sensation virtuelle, mélange de visuel, de sonore et de tactile [...] l'une des plus puissantes propriétés de l'interaction homme-machine ${ }^{13}$.

19 Selon l'analyse de ce game designer, un game feel réussi tient à une recette mélangeant cinq ingrédients principaux: la prise en compte des entrées (inputs) du joueur, et notamment l'importance des appareils d'interfaçage (manettes, claviers, etc.), et des commandes proposées (boutons, joystick, etc.) ; la qualité de la réponse apportée par la machine de computation qui s'accorde aux ordres donnés selon un panel très différent de possibles (voir le saut original de Mario, qui peut changer de direction en plein vol!) et là encore avec un usage additionnel des interfaces de sortie (son stéréo, manette vibrante, etc.) ; le contexte mondain dans lequel l'avatar du joueur évolue, sa richesse, l'affordance des objets qu'on y trouve, le type d'obstacles et de défis qu'ils dessinent; le polissage du monde et des actions exécutables, non seulement dans le rendu esthétique de l'œuvre (visuel et sonore), mais aussi par le choix des effets de réel proposés (e.g., 
des étincelles produites lors d'un coup porté sur certains matériaux); enfin, l'ancrage métaphorique choisi pour donner du sens à la simulation vidéoludique, son univers narratif $^{14}$ (une même mécanique de jeu, Rockstar Games nous l'a démontré éhontément, peut se déployer dans les rues d'une ville moderne motorisée, dans le désert accidenté d'un Far West crépusculaire, ou selon les codes d'un polar se déroulant dans le Los Angeles de 1947). Les principales expériences décrites comme répondant à cette notion de game feel sont : la sensation jouissive de contrôle, le plaisir de parfaire son adresse, l'extension de ses sens, l'extension de son identité ou l'interaction avec une réalité physique pleine et entière ${ }^{15}$.

L'art du game feel est difficile et les jeux qui marquent sont ceux qui savent trouver leur martingale. Cette notion pose à la fois la spécificité du médium vidéoludique, son enjeu crucial et ce qui va être la base sensationnelle pour créer une œuvre non plus expressive, mais inspirante.

20 À cette couche sensitive, le créateur va alors pouvoir tenter d'adjoindre une couche affective. Maîtrisant les rouages qui produisent une sensation dans le joueur, il pourra essayer de les décliner afin de générer des sentiments particuliers chez l'interacteur. À partir du moment où le joueur est im-pliqué ("plier, enrouler dans ») dans l'œuvre, intriqué dans sa trame et son devenir; il doit pouvoir laisser le sens de celle-ci l'inspirer (" souffler à l'intérieur de lui ») et l'é-mouvoir (« le sortir de son état premier»), selon une modalité presque sensuelle et intime. Cette inspiration des affects nous semble engendrée par l'entrelacement de trois approches.

21 La première est esthétique, c'est elle qui insiste sur l'immersion du joueur dans son univers de jeu, on pourrait l'appeler l'ambiance. Créer une ambiance de jeu, originale et captivante, n'est bien sûr pas qu'une question de moyens ni de résolution d'image. Certes, la tendance actuelle à proposer des jeux permettant de déambuler dans des environnements aussi réalistes que magnifiques, où chaque son, chaque texture, chaque ombre semble avoir été designé dans un but contemplatif et poétique, comme pour extraire l'avatar de son rôle d'aventurier et faire redécouvrir la beauté et la quiétude au joueur surstimulé, montre à quel point cette dimension est au cœur des problématiques d'immersion. Cependant, des jeux plus stylisés ne sont pas moins valeureux, comme le prouvent le noir et blanc de Limbo (Playdead, 2010), le pastel sombre d'Oxenfree (Night School Studio, 2016), le monde aquatique d'Abzû (Giant Squid, 2016), le dessin léger de Child of light (Ubisoft Montréal, 2014) ou le minimalisme de Return of the Obra Dinn (Lucas Pope, 2018) - sans parler des jeux d'horreur dont l'âme (et la réussite) se résume souvent à cette question d'ambiance. L'inventivité du game play (véritablement « mise en jeu », comme on parle de " mise en scène » au cinéma) est un ressort important de cette mise en disposition du joueur, en proposant au joueur des séquences de manipulation reflétant l'action en cours. Recréer une sensation, s'interroger sur "l'effet que fait ce que je fais », plonger le joueur dans un univers incroyable ou inapprochable (l'espace infini, les profondeurs océaniques): telle est l'ambition de bien des artistes modeleurs d'univers visuels ou sonores ${ }^{16}$.

La deuxième approche est narrative, elle s'évertue à créer l'empathie du joueur pour son avatar et les autres protagonistes, elle tourne autour de la genèse d'une émotion. Elle vise à produire un moment d'intimité forte avec le joueur, le voir pris non seulement aux tripes mais au cœur, lui arracher une larme qui ne serait pas vulgaire, ou lui faire simplement éprouver un sentiment naissant qui le change. On sait comment la théorie des neurones miroirs explique la sympathie que nous ressentons quand nous voyons un 
autre être vivre une situation donnée (les zones de notre cortex s'animent comme si nous éprouvions nous-mêmes la situation), mais ici la sympathie se mue en empathie car les actions de notre personnage ne sont pas celles d'un autre mais bien les nôtres ${ }^{17}$. Les scènes domestiques de Heavy Rain (Quantic Dream, 2010) sont touchantes et faites pour impliquer toujours plus le joueur dans la vie de ses avatars; les vies fragmentaires qui se devinent dans Dear Esther (The Chinese Room, 2012) ou dans What Remains of Edith Finch (Giant Sparrow, 2017) touchent plus que bien des romans; les jeux de Fumito Ueda sont peut-être parmi les premiers à avoir travaillé les relations entre l'avatar et un protagoniste (cheval, princesse, créature ailée), afin qu'elles aient une répercussion sur l'émotion du joueur.

La troisième approche est dramatique, elle consiste à responsabiliser le joueur vis-à-vis des actes de son avatar, elle organise la liberté créatrice du joueur. Le joueur s'implique parce qu'il est concerné par l'avenir de son avatar et de son aventure - et il l'est d'autant plus qu'il constate à quel point ses choix influent sur le déroulement du jeu. Se sentant libre de créer ses propres chemins dans le territoire virtuel du jeu, il se trouve responsable du devenir de ses protagonistes. Cela va bien au-delà de la séquence d'actions propres au franchissement d'un obstacle, cela concerne les choix moraux, sociaux ou dramatiques que le joueur prend et qui, dans les jeux les plus riches, s'avèrent lourds de conséquences. Les visual novels proposent souvent un choix en fin de dialogue pour réorienter le discours selon la préférence du joueur, qui sait qu'il touche là, chaque fois, à un embranchement subtil le menant à des ports différents (car il n'y a pas forcément de «bon" port, simplement des cheminements distincts qui se concluront diversement). Life Is Strange (Dontnod Entertainment, 2015) illustre exemplairement cette mise en responsabilité du joueur, l'avatar pouvant remonter le cours du temps afin de changer un destin fatidique et réécrire une nouvelle histoire, la sienne.

Le tout concourt bien, et de façon de plus en plus subtile et réussie, à faire naître des sentiments puissants chez le joueur impliqué. Une tendance récente dans le jeu vidéo en est peut-être le fer de lance, celle des jeux autobiographiques, celle que le théoricien et game designer Sébastien Genvo appelle les "jeux expressifs ». "Le concept de jeu expressif renvoie à un jeu proposant au joueur de se mettre à la place d'autrui pour explorer ses problèmes psychologiques ou sociaux, et ainsi de faire l'expérience des dilemmes moraux et éthiques qui en résultent ${ }^{18} »$. Or elle irrigue bien plus largement. Le jeu The Path (Tale of Tales, 2009), par exemple, plonge le joueur dans une leçon de vie onirique, lourde de fantasmes larvés : contrôlant successivement six avatars plus ou moins âgés du chaperon rouge, le joueur ne se frotte à la vie qu'en désobéissant à l'ordre explicite du jeu ("restez sur le chemin»), rencontrant des loups à la figure changeante et explorant ainsi une facette du devenir-femme.

\section{La pensée modulaire}

S'il est bien un acte de création, le geste du game designer ne relève pas d'une pensée plastique $^{19}$ telle que les beaux-arts nous ont habitués à la comprendre. Celle de l'artiste plasticien est une pensée équivoque, en actes et en corps, où la matière s'affronte physiquement, où l'accident survient, et qui se déploie selon les failles du médium et l'intimité du corps créateur; elle est foncièrement physique, inexplicable, non schématisable ${ }^{20}$. La pensée créatrice que la technologie amène est algorithmique : elle 
tient tout entière en ses schémas, l'étalement de ses procédures, la symbolisation codée de tout son spectre d'apparition, son dess(e)in synoptique ${ }^{21}$.

On pourrait encore préciser le type de pensée créatrice à l'œuvre dans le jeu vidéo en la caractérisant comme une pensée hyperanalytique, et même modulaire, puisqu'elle travaille souvent par fonctions, traitant des modules de données les uns après les autres. Comment se déroule le travail de conception du créateur vidéoludique? Que fait-il ? Quelles étapes suit-il ?

Le game designer travaille par strates successives, concentriques, s'appliquant chaque fois sur un aspect fonctionnel ou mondain du jeu à venir. Tel narrative designer ${ }^{22}$ nous expliquait qu'il s'appliquait à travailler par strates : d'abord la mécanique de jeu, puis l'univers dans lequel elle va s'inscrire, puis le type de personnages qui le peuplent et enfin l'histoire qui va s'y dérouler. C'est ainsi qu'un jeu se conçoit, comme un organisme fait d'un squelette, d'un réseau sanguin, de nerfs, de muscles, etc. Il s'agit toujours de revenir sur le métier pour ajouter une nouvelle couche fonctionnelle. Photoshop a habitué les artistes numériques à travailler par calques. C'est cette technique qui prévaut toujours: dans notre cas, chaque couche est fonctionnelle, participant à la production de travaux toujours plus fouillés et denses. Notons que cette approche est autant épi- que phylogénétique : l'histoire du jeu vidéo montre que ces mêmes couches se sont aussi ajoutées au fur et à mesure de la maturation du domaine du jeu vidéo.

Le game designer travaille en proposant un florilège d'options, à valider ou à choisir. Les possibles de l'artiste vidéoludique sont sans limite a priori ${ }^{23}$, mais chacun a un coût et un intérêt propre. Il est aussi important de savoir ce que contiendra le jeu que de trancher sur ce qu'il ne contiendra pas. Combien de races différentes peupleront l'univers? Quels types d'objets seront disponibles, quels niveaux de détail visuel seront les leurs et quelles fonctionnalités déclencheront-ils? Les logiques propres à l'œuvre seront ici rejointes par des logiques technologiques et économiques pour élire les options retenues.

28 Le game designer travaille en proposant une histoire en miettes, une histoire contée par bribes, une histoire à disséminer en lambeaux. En cela, le travail du scénariste de jeu vidéo n'a que peu à voir avec celui de l'écrivain. Son histoire se déploie selon divers procédés : action, image, son, texte. Il doit donc exploser son histoire dans autant de briques de nature différente. Quant au texte, il est lui-même éparpillé entre différents vecteurs narratifs (voix hors champ, panneaux signalétiques, objets informatifs, dialogues) qui distribuent les bonnes informations, souvent changeantes, en fonction du déroulement de l'histoire et des actions du joueur. Le récit s'écrit dans une immense matrice numérique qui recense chaque bribe d'histoire, son lieu d'apparition et ses différentes options possibles. En jeu vidéo, « en grande partie, l'écriture est avant tout une distribution d'informations ${ }^{24} »-$ autant pour les romanciers et les esthéticiens ${ }^{25}$ ! L'axe syntagmatique de l'écriture se voit explosé.

Le game designer travaille en proposant un maquis foisonnant d'histoires. Chaque portion de scénario est elle-même le réceptacle d'options variées. Les dialogues, pour ceux qui offrent au joueur de réelles possibilités de choix, sont tout de suite exponentiels. Ne s'écrit pas seulement l'histoire la plus probable, mais plusieurs histoires possibles. Que se serait-il passé si Cyrano avait refusé d'aider Christian à conquérir sa belle? Ou s'il avait avoué à Roxane la supercherie dès la scène du balcon ? C'est tout cela que le scénario vidéoludique permet d'interroger et de vivre, et pas 
seulement la version de Rostand. Il n'y a pas, à proprement parler, une histoire, mais plutôt un faisceau de récits différents pris dans une trame plus ou moins prédéterminée. L'axe paradigmatique de l'écriture est démultiplié.

Enfin, le travail de conception du jeu vidéo progresse de manière itérative. Les itérations sont essentielles à la production: échanges d'idées entre les différents acteurs, validations, tests, réécriture, amendements, nouveaux tests. La forme générale de la conception est celle d'une suite indéfinie d'itérations, jusqu'à ce que, de guerre lasse, en manque de fonds, ou par choix positif, un arrêt définitif de la séquence soit proposé. Pas si définitif que cela puisqu'il pourra être remis en cause par un travail ultérieur sur un autre objet à concevoir - afin d'obtenir in fine un résultat cohérent.

La pensée créatrice dans le jeu vidéo est donc discrète et fonctionnalisée, émiettée voire granulaire, disséminée et "disséminante", cartographiable (l'usage des mind maps y est traditionnel), entièrement explicite, univoque, sans arrière-fond, sans indicible et sans impalpable. Le corps créateur n'y a pas sa place, l'esprit algorithmisé l'a remplacé. L'individu créateur y est l'exception, le groupe spécialisé mis en réseau doit pouvoir assurer une plus grande efficacité pour un résultat impeccable; car le créateur vidéoludique chasse l'erreur, pas le plasticien. Celui-ci peut la tolérer, la retourner, voire la magnifier - celui-là la traque et veut abolir sa version inacceptable : le bug.

L'une des figures les plus répandues de l'iconographie orthodoxe est celle du Christ pantocrator: on y voit le buste du Christ, une main posée sur les Évangiles, l'autre main levée en signe de bénédiction ou d'enseignement. Ce n'est pas un Christ en actes, sorte d'artiste furieux s'affrontant à la matière de sa création, mais un Christ immobile, animé de la seule puissance du verbe et de l'écrit, créant par le symbole. Le game designer crée par le code et les appareils. Panto-krator veut dire puissance de tout, omnipotence de création sur le monde qu'il a conçu - tel pourrait être l'idéal numérique du créateur de jeu vidéo...

\section{BIBLIOGRAPHIE}

Adorno Theodor W., Théorie esthétique, trad. de l'allemand M. Jimenez et E. Kaufholz, Paris, Klincksieck, coll. « Esthétique », 1995.

Alain, «Vingt leçons sur les Beaux-arts », in Les Arts et les Dieux, éd. établie et présentée par G. Bénézé, préface A. Bridoux, Paris, Gallimard, coll. « Bibliothèque de la Pléiade », 1958.

Aristote, La Métaphysique, éd. établie par J. Tricot, Paris, Vrin, coll. « Bibliothèque des textes philosophiques », 1970.

Baron Sean, « Cognitive flow: the psychology of great game design », in Gamasutra, 22 mars 2012. URL : https://www.gamasutra.com/view/feature/166972/ cognitive_flow_the_psychology_of_php [consulté le 01/03/2021]

Bogost Ian, Persuasive Games: The Expressive Power of Videogames, Cambridge, The MIT Press, 2007. 
Chen Jenova, « Le flow dans les jeux » [en ligne], mémoire de master, trad. de l'anglais B. Swales, Université de Californie du Sud, 2006. URL : http://jenovachen.com/flowingames/

Flow_in_games_final_FRENCH.pdf[consulté le 01/03/2021]

Couchot Edmond, La Nature de l'art : ce que les sciences cognitives nous révèlent sur le plaisir esthétique, Paris, Hermann, 2012.

Csíkszentmihályi Mihály, Flow: The Psychology of Optimal Experience, Harper Perennial, coll. « Modern classics », 1990.

Farges Julien, « Monde de la vie et philosophie de la vie. Husserl entre Eucken et Dilthey », Études Germaniques [en ligne], $\mathrm{n}^{\circ}$ 242, p. 191-217. URL : https://www.cairn.info/revue-etudesgermaniques-2006-2-page-191.htm\#re7no7 [consulté le 01/03/2021]

Francastel Pierre, Art et technique aux XIX et XX siècles, Paris, Gallimard, coll. « Tel », 1988.

Fried Michael, La Place du spectateur. Esthétique et origines de la peinture moderne, trad. de l'anglais C. Brunet, Paris, Gallimard, coll. « NRF Essais », 1990.

Genvo Sébastien, « \#22 L'expressivité dans les jeux vidéo - Synthèse », in Expressive Game : Plateforme d'analyse et de création de jeux vidéo, 05 janvier 2021. URL : http:// www.expressivegame.com/publications/type/expressivite-jeux-video-synthese/ [consulté le 01/03/2021]

Goodman Nelson, Manières de faire des mondes [1978], trad. de l'anglais M.-D. Popelard, Nîmes, Jacqueline Chambon, coll. « Rayon art », 1992.

Hunicke Robin, Leblanc Marc et Zubek Robert, « MDA: a formal approach to game design and game research », in Dan Fu, Stottler Henke et Jeff Orkin (dir.), Challenges in game artificial intelligence, San José, 25-26 juillet, Menlo Park, American Association for Artificial Intelligence, 2004. URL : https://aaai.org/Papers/Workshops/2004/WS-04-04/WS04-04-001.pdf [consulté le 01/03/2021]

Koster Ralph, A theory of fun for game design, avant-propos W. Wright, Sebastopol, O'Reilly Media, 2013.

Krajewski Pascal, L'Art au risque de la technologie, Paris, L'Harmattan, coll. « Ouverture philosophique », 2013.

Krajewski Pascal (dir.), « Ars Ludens - arte, jogo e lúdico » [en ligne], Revista de Ciências da Arte, $\mathrm{n}^{\mathrm{o}}$ 6-7, 2019. URL : http://convocarte.belasartes.ulisboa.pt/index.php/2019/04/03/n-o-6-e-n-o-7ars-ludens-arte-jogo-e-ludico/ [consulté le 01/03/2021]

Lubie Lou, «Écrire un scénario de jeu vidéo : Les conseils d'un professionnel », in Le forum dessiné, 2013. URL : https://www.forum-dessine.fr/tutoriels/ecrire-un-scenario-de-jeu-video [consulté le 01/03/2021]

Marin Louis, De la représentation, recueil établi par D. Arasse et al., Paris, École des hautes études en sciences sociales, Gallimard, Seuil, coll. « Hautes études », 1994.

Space Game Studio, « MOTHERSHIP prototype [Concept trailer] », in Youtube, 22 octobre 2020. URL : https://www.youtube.com/watch?v=S3wVzCLfXD8 [consulté le 01/03/2021]

Swink Steve, Game feel : a game designer's guide to virtual sensation, Burlington, Morgan Kaufmann Publishers, 2009.

Swink Steve, «Game feel : the secret ingredient », in Gamasutra, 23 novembre 2007. URL : https:// www.gamasutra.com/view/feature/2322/game_feel_the_secret_ingredient.php [consulté le 01/03/2021] 


\section{NOTES}

1. Nelson Goodman ne dit pas autre chose, même s'il rajoute une troisième composante : « Un art sans représentation ou expression ou exemplification - oui ; un art sans les trois à la fois - non ». Nelson Goodman, "Quand y a-t-il art?", in Manières de faire des mondes [1978], Nîmes, Jacqueline Chambon, coll. « Rayon art », 1992, p. 99.

2. Theodor Adorno, Théorie esthétique, Paris, Klincksieck, coll. « Esthétique », 1995, p. 152.

3. «Représenter signifie se présenter représentant quelque chose et toute représentation, tout signe ou procès représentationnel comprend une double dimension - dimension réflexive, se présenter; et dimension transitive, représenter quelque chose - et un double effet : l'effet de sujet et l'effet d'objet ». Louis Marin, "Mimésis et description », in De la représentation, Paris, École des hautes études en sciences sociales, Gallimard, Seuil, coll. « Hautes études », 1994, p. 255.

4. Theodor Adorno, op. cit., p. 161 et p. 163.

5. Et non de l'artiste ou du spectateur. Nelson Goodman, op. cit., p. 116.

6. Pascal Krajewski, «De la synthétisation à la synthèse figurale», in L'Art au risque de la technologie, t. I Les Appareils à l'œuvre, Paris, L'Harmattan, coll. « Ouverture philosophique », 2013, p. 115-128.

7. Plutôt au sens de Schein, simulacre, ici.

8. La dynamis est l'être-en-puissance. Elle est : 1 / le principe du mouvement ou du changement dans un autre être ou dans le même être en tant qu'autre ; 2 / le pouvoir pour un patient de recevoir une modification quelconque ; $3 /$ la faculté de mener quelque chose à bien. En bref : le pouvoir effectif de changer ou d'être changé. L'energeia est l'être-en-acte; toute chose mue ou motrice est un être-en-acte. Aristote, "Livres $\Theta$ et $\Delta$ ", in La Métaphysique, Paris, Vrin, coll. «Bibliothèque des textes philosophiques », 1970.

9. Julien Farges, "Monde de la vie et philosophie de la vie. Husserl entre Eucken et Dilthey ", Études Germaniques [en ligne], $\mathrm{n}^{\circ} 242$, p. 191-217. URL: https://www.cairn.info/revue-etudesgermaniques-2006-2-page-191.htm\#re7no7 [consulté le 01/03/2021]

10. Theodor Adorno, op. cit., p. 161.

11. Pascal Krajewski, «L'espace des archétypes », in L'Art au risque de la technologie, op. cit., p. 152-181.

12. Elle nous semble notamment intégrer les approches concernant le «flow ", théorisé par le psychologue Mihály Csíkszentmihályi, cet art de mener le joueur sur un chemin pentu mais non escarpé, à mi-chemin entre l'ennui et l'anxiété, mesurant voire ajustant la difficulté aux compétences du joueur, visant le plaisir du jeu et la satisfaction de la réussite ; ou même celles du "fun », que Ralph Koster analyse comme le plaisir produit par la maîtrise mentale d'un problème et qui passe donc par un processus d'apprentissage construit sur trois piliers: des feedbacks (retours d'information) variables (non prévisibles), un parcours devant mener le joueur à l'excellence et une gestion constructive des échecs.

13. Nous traduisons : Steve Swink, Game feel : a game designer's guide to virtual sensation, Burlington, Morgan Kaufmann Publishers, 2009, p. XIII.

14. Steve Swink, "Game feel: the secret ingredient ", in Gamasutra, 23 novembre 2007. URL : https://www.gamasutra.com/view/feature/2322/game_feel_the_secret_ingredient.php

[consulté le 01/03/2021]

15. Steve Swink, Game feel : a game designer's guide to virtual sensation, op. cit., p. 10-12.

16. Ainsi du projet Mothership, développé par Carlos Botella, qui vise notamment à nous plonger de manière réaliste au cœur d'une station spatiale explorant l'univers. Space Game Studio, Space Game Studio, «MOTHERSHIP prototype [Concept trailer] », in Youtube, 22 octobre 2020. URL : https://www.youtube.com/watch?v=S3wVzCLfXD8 [consulté le 01/03/2021] 
17. Pour une approche artistique de la théorie des neurones miroirs, voir : Edmond Couchot, La Nature de l'art: ce que les sciences cognitives nous révèlent sur le plaisir esthétique, Paris, Hermann, 2012.

18. Sébastien Genvo, "\#22 L'expressivité dans les jeux vidéo - Synthèse », in Expressive Game: Plateforme d'analyse et de création de jeux vidéo, 05 janvier 2021. URL: http:// www.expressivegame.com/publications/type/expressivite-jeux-video-synthese/ [consulté le 01/03/2021]

19. Pierre Francastel, Art et technique aux XIX ${ }^{e}$ et XX $X^{e}$ siècles, Paris, Gallimard, coll. « Tel », 1988.

20. Alain, "Leçon 2 ", in "Vingt leçons sur les Beaux-arts", in Les Arts et les Dieux, Paris, Gallimard, coll. «Bibliothèque de la Pléiade », 1958, p. 480 et suivantes.

21. Pascal Krajewski, "L'espace des archétypes", in L'Art au risque de la technologie, op. cit., p. $152-181$.

22. Tout ce passage doit beaucoup à l'entretien que nous a accordé Léonard Bertos, écrivain, scénariste et narrative designer.

23. À cette heure, ce constat semble presque acquis, même si ce ne fut pas le cas par le passé. On connaît bien des cas de jeux vidéo qui durent attendre la génération de consoles suivantes pour trouver dans leurs nouvelles puissances computationnelles les moyens de mettre en jeu leur projet (c'est le cas d'Ico de Ueda).

24. Olivier Henriot, expert scénario chez Ubisoft, cité dans : «Écrire un scénario de jeu vidéo : Les conseils d'un professionnel », in Le forum dessiné, 2013. URL: https://www.forum-dessine.fr/ tutoriels/ecrire-un-scenario-de-jeu-video [consulté le 01/03/2021]

25. "Aucune œuvre d'art ne doit être décrite ni expliquée sous les catégories de la communication ». Theodor Adorno, op. cit., p. 158.

\section{RÉSUMÉS}

Si le jeu vidéo est bien un nouveau médium artistique, il tire ses spécificités du régime d'interaction qu'il noue avec son spectateur et de la place particulière qu'il lui octroie dans le déploiement de l'œuvre. Dès lors, les enjeux artistiques de la création vidéoludique devront s'analyser non plus comme un travail autour de la représentation et de l'expression, mais selon leur versant technologique, à savoir la simulation et l'inspiration. Ces deux nouvelles voies visent respectivement à inventer des mondes habités que le joueur peut expérimenter et à lui insuffler de véritables émotions en se focalisant sur l'effet induit par le système et l'univers du jeu. Le nouvel artiste créateur ne se retrouve plus dans la notion de pensée plastique mais met en œuvre une nouvelle forme de pensée créatrice, toute numérique et réticulaire, algorithmique, modulaire. Le game designer se rêve artiste pantocrator, tout puissant, capable de tout créer par la seule puissance du code et des appareils.

\section{INDEX}

Mots-clés : interaction, simulation, inspiration, représentation, expression, pensée plastique, algorithme 


\section{AUTEUR}

\section{PASCAL KRAJEWSKI}

Docteur en sciences de l'art, Pascal Krajewski est chercheur associé à la faculté des beaux-arts de l'université de Lisbonne (laboratoire Cieba). Son travail de recherche porte sur l'art des nouveaux médias, la technologie, les appareils, les images, la bande dessinée. Il est l'auteur de plusieurs ouvrages, dont L'Art au risque de la technologie (2013), L'Ordre technologique (2016) et Art, médium, média (2018). 